\title{
e-Phaïstos
}

e-Phaïstos

Revue d'histoire des techniques / Journal of the history

of technology

V-1 2016 | 2018

Ville et technique

\section{Technologies numériques et durables aux défis de la métropole sensible}

Technological and sustainable cities issues for senseable metropolis

\section{Émeline Bailly}

\section{(2) OpenEdition}

Journals

Édition électronique

URL : http://journals.openedition.org/ephaistos/1309

DOI : 10.4000/ephaistos. 1309

ISSN : 2552-0741

Éditeur

IHMC - Institut d'histoire moderne et contemporaine (UMR 8066)

\section{Référence électronique}

Émeline Bailly, «Technologies numériques et durables aux défis de la métropole sensible », e-Phaïstos [En ligne], V-1 2016 | 2018, mis en ligne le 28 janvier 2018, consulté le 19 avril 2019. URL : http:// journals.openedition.org/ephaistos/1309 ; DOI : 10.4000/ephaistos.1309

Ce document a été généré automatiquement le 19 avril 2019

Tous droits réservés 


\title{
Technologies numériques et durables aux défis de la métropole sensible
}

Technological and sustainable cities issues for senseable metropolis

\author{
Émeline Bailly
}

\section{Introduction}

1 Ville durable, sobre, dense et ville numérique, virtuelle, connectée,

intelligente, ne constituent-elles pas un nouvel idéal de ville technologique ou au contraire une invitation à penser l'urbain selon un angle nouveau ?

La conception urbaine a souvent évolué grâce aux avancées techniques, c'est-à-dire aux précédés, méthodes et savoir-faire permettant d'améliorer l'environnement humain. La maîtrise agraire et la canalisation de l'eau ont permis l'édification des premières villes,

l'édification des routes, leurs mises en réseaux commerciales, l'industrialisation, le développement de la ville moderne (rails, infrastructure, zoning, etc.). Ainsi, la ville technique, liée à des finalités particulières, est devenue technologique par la complexité

des techniques qui s'entremêlent. 
Aujourd'hui, les technologies environnementales et numériques s'établissent au nom de la ville durable. Or, avec le développement durable, l'ambition technique, en particulier environnementale et numérique, vise au contraire à préserver la planète, ses ressources, la vie humaine et les civilisations. La technologie change de statut. Elle n'est plus pour le progrès de l'homme, mais pour la pérennité du monde dont l'homme doit dorénavant prendre soin. Comment, dès lors, ce renversement de paradigme impacte-t-il la culture technique héritée et la conception urbaine actuelle ? En quoi ces techniques environnementales visant la maîtrise d'énergie, de l'eau, la protection des ressources et celles numériques visant la création de réseaux intelligents, de nouveaux services

urbains, d'information en continue, permettent-elles de concevoir une ville durable? Proposent-elles (ou non) une nouvelle approche urbaine au moment où les grands projets

. Il s'agira de comprendre ce qui fonde la ville durable et numérique pour les politiques publiques afin de questionner les idéaux technologiques urbains associés. Après une analyse de la mobilisation des technologies dans les discours actuels des professionnels de la ville, nous questionnerons la possibilité d'un changement de paradigme de la technique pour des métropoles au service de la durabilité de la planète mais aussi offrant des expériences à la fois sensibles et objectives des lieux urbains.

De fait, la ville des avancées technologiques ne peut-elle être au service de la préservation de notre milieu de vie et de ce monde sensible qui la traverse ? L'appréhension du monde sensible qui nous environne a été délaissée au profit des approches rationnelles. Or, ce monde sensible nous envahit. Nous le vivons avec nos sens, nous le ressentons avec nos sentiments et émotions, à défaut de l'objectiver. Il nous semble dès lors que les technologies qui se mêlent aux lieux de vie peuvent participer du monde sensible, c'est-àdire des systèmes d'affects (sensations, sentiments et émotions) et d'imaginaires qui

\section{Ville durable, ville numérique, nouveau paradigme du progrès technique?}

\section{La métropole, nouveau milieu des hommes}

Si la ville s'est déployée grâce à une succession de progrès techniques, la métropolisation est aujourd'hui plus que jamais liée aux innovations technologiques, notamment de l'information et télécommunication (NTIC). La compétition des villes- mondes se joue par la « concentration métropolitaine ", non seulement des hommes, des capitaux et infrastructures mais aussi des innovations (Saskia Sassen, 1991). La possibilité d'inventer des nouvelles techniques est devenue un enjeu économique, mais aussi de la métropolisation. 
La métropole résulte d'un ensemble de processus où les réseaux sont étroitement imbriqués avec des facteurs géopolitiques et urbains marqués par la globalisation

. Elle favorise les sociétés de réseaux, avec la circulation de l'information et l'essor du

$»^{3}$. Elle conduit à l'émergence deglobal cities

« cyberurbain au centre des pouvoirs économiques mondiaux, telles Londres, New York, Paris ou Tokyo ${ }^{4}$ . Elle reconfigure l'équilibre géo-urbain mondial et conduit à des formes d'extension et de densification urbaines sans précédent (plus de la moitié de l'humanité est devenue urbaine).

Chaque mégalopole est connectée aux autres, aux banques, grandes firmes internationales, aux boutiques franchisées, aux mouvements militants. Cette nébuleuse fait naître un sentiment du monde globalisé, un paysage sans commune mesure avec le monde connu des hommes. Cette croissance urbaine sans limite annonce la fin de la ville. Elle est pourtant remise en cause par le risque qu'elle représente pour le devenir humain : disparition des terres agricoles, pollutions, épuisement des ressources, perte de biodiversité, etc.

De fait, pour les modernistes, avec le progrès technique, l'homme s'était affranchi de la nature. L'extension urbaine récente était dans cette conception, une domination de l'homme sur l'environnement. Elle fut mise à mal par les problèmes écologiques et sociaux des années 70 , (pollutions, nuisances sonores, fragmentation sociale et territoriale, territoire de relégation avec les quartiers informels, etc.). La préoccupation environnementale est alors portée au niveau international avec une série de congrès et déclarations en faveur de la protection de la nature (Kyoto, Rio, rapport Brundtland

) et au niveau national par la mise en place de politiques environnementales. En France, par exemple, le ministère de l'Environnement, créé en 1971, a pour mission de protéger les équilibres ruraux/urbains et les espaces naturels (parcs régionaux). La protection des territoires est introduite

. La nature et les biens environnementaux deviennent une ressource à préserver, un bien commun. Avec la métropolisation, l'investigation technique environnementale mais aussi numérique apparaît alors comme la réponse aux maux des métropoles, devenues le milieu des hommes. 


\section{Ville durable ou l'idéal de la ville technico- environnementale}

Les enjeux de la ville durable visent, à travers le développement durable, à préserver la planète et maîtriser l'urbanisation continue du monde. Les enjeux de technologies urbaines environnementales se sont d'abord centrés sur la réduction des énergies des bâtiments (Bâtiment Bas Carbone, Bâtiment à Energie Positive, etc.) mobilisant des techniques de protections thermiques, de chauffage solaire, etc. Parallèlement, d'autres mesures se sont déployées autour de la gestion de l'eau, de la création de trames de biodiversité, les mobilités douces (tramway, pistes cyclables, etc.) pour n'en décrire que quelques éléments marquants.

Les techniques environnementales sont mobilisées pour limiter les impacts néfastes de l'urbanisation pour la survie de l'espèce humaine. Elles sont convoquées pour corriger les effets écologiques de la poursuite de la croissance économique et urbaine. Elles s'imposent comme une nouvelle norme urbaine. Ces dispositifs ou plutôt objets technicoenvironnementaux deviennent aussi des emblèmes d'une image urbaine renouvelée. Par

exemple, en matière de déplacement, après des décennies d'urbanisme centré sur les réseaux viaires, la recherche d'alternatives aux véhicules polluants et énergivores met à l'honneur les villes des transports en commun et déplacements doux (à vélo, à pied). Du tout voiture, les politiques urbaines plébiscitent à présent de nouvelles infrastructures et modes de déplacement plus propres afin de désengorger le trafic automobile, limiter les pollutions et donner une image plus « saine ». De même, au nom de la sobriété

énergétique, la conception et l'implantation des bâtiments réorganise la composition urbaine (urbanisme en peigne, orientation nord sud, élargissement de voies pour garantir

l'ensoleillement au sud, venelle et façade aveugle en double peau au nord, etc.). On pourrait multiplier les exemples des implications environnementales sur la fabrique de la

Ces choix reconfigurent les métropoles et leurs modèles urbains au nom de la protection de l'environnement mais aussi de la compétition économique. Ils conduisent à renouveler

la fabrique urbaine mais aussi en conséquence les manières de vivre ces espaces. Pour autant, l'évolution des modes de vie reste peu abordée dans les discours de professionnels. Ceux-ci masquent leur absence de vision, par des objectifs technico environnementaux, devenus emblème de la ville durable. Il s'ensuit une série de formules abstraites censées traduire la ville environnementale. Ville sobre, ville dense, ville nature,

ville viable, etc. sont autant de formules qui masquent un impensé urbain. Sans plus de vision, c'est la maitrise technique des risques de l'urbanisation qui guide les projets urbains à laquelle les hommes doivent s'adapter. 


\section{Ville numérique ou l'idéal de ville des technologiques de l'information}

La ville numérique, c'est-à-dire ayant recours aux technologies de l'information (wifi, réseaux intelligents, services liés à des informations en temps réels, applications sur smartphone...) se déploie de manière plus récente. Elle n'existe pas en soi, étant le plus souvent associée à des dispositifs existants. Elle apparaît comme un attribut qui vient enrichir des éléments du réel (réseaux, lieux collectifs, réseaux de voirie, de transports, etc.). Elle apparaît moins englobante que le développement durable. Par exemple, les infrastructures de flux numériques doublent les réseaux physiques pour les doter de nouvelles capacités (performance énergétique de l'éclairage public, prévisions des flux, réseaux communicants afin de prévoir les dysfonctionnements et renseigner les utilisateurs, tels les embouteillages, etc.).

Avec la géolocalisation, les données virtuelles envahissent l'espace urbain, augmentant continuellement d'informations les métropoles. Cette connaissance nouvelle des milieux crée des fonctionnalités nouvelles mais aussi des systèmes de données cohérents, intelligents et prédictifs ( data city

). La ville numérique est d'ailleurs décrite en fonction des différentes technologies de l'information. On parle de « ville intelligente », de « smart city

» optimisée par des systèmes intelligents de collecte, partage, rediffusion de l'information 7

mais aussi de ville connectée (accessible aux réseaux de l'information), ou virtuelle, notamment à travers les maquettes numériques urbaines (MNU). Les urbanistes et collectivités ont un usage croissant des représentations 3D, surtout comme outil de promotion des futurs quartiers aménagés (marketing urbain) ou d'information/ consultation des habitants. Plus rarement, ces dernières sont mobilisées pour modéliser l'impact des aménagements projetés, notamment environnementaux (bruit, risques naturels, ensoleillement, exposition au vent, etc.) ou comme outil collaboratif entre professionnels (monitoring, etc.) ou entre habitants et élus. 
La technologie numérique est censée améliorer les capacités, ou " performances » de la ville mais elle tend aussi à en favoriser la distanciation, au profit des mondes de flux, de réseaux parallèles, dont la rapidité et l'efficience sont considérées essentielles à la compétition mondiale des métropoles. Elle crée un monde infini, globalisé, en échange permanent. Elle étend l'espace qui se dématérialise en partie, même si chaque objet technique reste lié à un objet urbain. Les chercheurs britanniques Andy Crabtree et Tom Rodden ont d'ailleurs fait une typologie des médiations numériques à l'œuvre

\section{Ils distinguent lesmedia spaces}

qui mettent en relation les lieux, les objets numériques incrustés dans l'espace physique, la réalité augmentée qui fusionne les mondes physiques et virtuels par des couches d'information, l'écologie hybride qui mêle les deux mondes alternativement, tels les jeux de rôle qui peuvent être sur le web et dans le réel, les smartphones, qui proposent un regard vers les monde (photo, orientation, choix, etc.) tout en offrant de nouvelles expériences sensibles. Ce sont autant de médiums techniques au service de la métropolisation et de la mondialisation économique et sociétale. D'une certaine manière, la société numérique crée des offres et des besoins nouveaux pour favoriser la croissance et la métropolisation comme réponses aux problèmes économiques et urbains.

\section{Ville durable, ville numérique, la technique au service de la pérennité et l'infini du monde... virtuel ?}

Avec la ville durable et numérique, la métropole est censée se développer vers plus de

fonctionnalités nouvelles tout en étant protégée des risques environnementaux et sociaux. Pourtant, le développement urbain durable se préoccupe surtout de techniques environnementales et moins des problématiques sociétales ou écologiques. De même, la

ville numérique crée des connaissances nouvelles, des innovations économiques, sans pour autant s'interroger sur l'évolution des modes de vies et besoins des usagers des métropoles. On assiste donc à un retour aux modèles urbains fabriqués avec des principes

d'intervention urbaine s'appliquant à tout territoire comme par exemple la charte d'Athènes. Or, ceux-ci avaient tendance à décliner ces dernières années au profit des

. Ils sont légitimés par un discours technico-urbain à même de se décliner localement au nom de sauvegarde de la planète.

Cette tendance techniciste est d'autant plus marquée qu'à présent une hybridation des deux visions de ville numérique et durable est à l'œuvre. En effet, presque toutes les innovations de la ville durable sont liées au numérique et vice versa. Des modes de déplacement doux sont connectés (vélos, voitures électriques partagées), des données informent des niveaux de pollution et incitent à des modifications des comportements (recours aux transports en commun par exemple), les arbres sont connectés de puces pour veiller à agir en continu sur leur entretien, etc. Les politiques publiques s'attachent de plus en plus à augmenter de qualité numérique et environnementale des espaces urbains sans pour autant s'interroger sur leur impact pour la vie des hommes. Or, les métropoles sont plus qu'un environnement technique. Elles sont le lieu de la vie humaine, 
Ainsi, la ville durable et numérique constitue un nouvel idéal de la ville technologique assemblée. Ce rapprochement fait que le numérique, n'est plus seulement un domaine

appliqué aux objets digitaux ou aux réseaux mais vient infuser toute la conception urbaine, englobant comme le développement durable tout ce qui fait l'espace urbain. Il vise autant l'essor économique que la préservation de la planète. Dans leur alliance, villes durable et numérique proposent une vision « solutionniste ", c'est-à-dire une propension à croire que la technologie peut résoudre tous les problèmes de l'humanité. Autrement dit, elle vise à réparer les progrès de l'homme devenu nuisible.

\section{Des normes techniques déconnectées des évolutions urbaines et sociétales}

Si les politiques urbaines s'ancrent dans une vision solutionniste, qu'en est-il du rapport des individus à la technique ? Plus qu'un changement de paradigme technique, n'est-on pas plutôt dans une évolution sociétale qui implique d'aborder autrement la technique et la métropole elle-même ? Notre hypothèse est que la technique pourrait être un levier pour accompagner le changement sociétal à l'œuvre, au-delà des problèmes écologiques, les crises économiques et sociales qui ont motivé son déploiement. En effet, Jérémy Rifkin

(2014), annonce une grande mutation économique du fait de la convergence de trois révolutions technologiques simultanées (énergie, communication et transports).

\section{Des politiques urbaines technico - normatives en mal de faire la ville autrement}

Les tenants du développement numérique et durable prônent des espaces urbains renouvelés qui ne sont pas sans contradiction. La ville durable remet en cause le mythe de

l'extension illimitée de la ville (lutte contre l'étalement urbain) alors que la ville numérique promet l'extension du monde urbain (réseaux de câbles numériques, maximisation des flux et interconnexions, etc.). Loin d'être affichées comme opposées, elles sont considérées comme complémentaires, la première favorisant une reterritorialisation, la seconde des communautés d'appartenances.

De fait, la ville durable (dense et compacte) et numérique (lien à distance) serait source de reterritorialisation à travers des formes de proximité nouvelles (espaces publics de nature, circuit court, e-commerce, télétravail...). Ainsi, parallèlement à la déterritorialisation des flux immatériels, le local serait réinvesti. Des centralités urbaines sont projetés autour d'espaces publics, équipements et commerces, hubs de transports en commun connectés en vue de créer des pôles urbains de proximité. Des métropoles multi

polaires semblent s'ériger, à la fois durables et augmentées numériquement. C'est un idéal vertueux qui s'inventerait, avec la limitation des déplacements, des dépenses 
Cette vision spatiale fait même l'objet d'une vision idéalisée du local. L'utopie numérique promet une communauté humaine affranchie de la globalisation économique. Internet apparaît comme une opportunité inédite pour inventer d'autres modèles d'être ensemble (partage de données, échange, solidarité, etc.). Il est envisagé comme un moyen pour l'individu de s'émanciper d'une société hiérarchique, bureaucratique et aliénante 10

. L'utopie durable promet, quant à elle, une vie de voisinage protégée des risques naturels, les déplacements doux (marche et vélo), les aménités du proche. Elle promeut une qualité de vie locale autour de valeurs de partage (énergie, eau, voiries, véhicules, etc.), de communauté (économie circulaire, sociale et solidaire etc.), de ressources communes (jardins partagés, recyclage, etc.). Ils appellent à reconsidérer les lieux vécus, investis, expérimentés localement, mais aussi leurs paysages que la ville des flux avait eu tendance

à mettre au second plan.

De fait, les discours des politiques urbaines durables ou numériques introduisent une ambition de lien entre la ville locale et l'intérêt global de la planète. Mais pour reterritorialiser l'hyper-espace abstrait et dématérialisé, marqueur du divorce nature/ culture, ne faudrait-il pas plutôt songer à une revalorisation des territoires comme produit de l'homme et constitués de lieux vécus, pratiqués et dotés d'identités propres 11

. La géolocalisation de la ville numérique mais aussi la naturalisation des écoquartiers ouvrent une possible voie vers ce retour aux lieux. Pour autant, cette préoccupation territoriale reste à l'état de discours d'intention. Il faut dire que la composition urbaine d'un grand nombre d'écoquartiers continue de s'ériger en périphérie des villes. Il privilégie les espaces collectifs privés au détriment des espaces publics. La proposition grandissante de macro-lots favorise le repli des ensembles résidentiels vers des espaces privés d'agréments en cœur d'îlot. De même, des espaces de nature, ayant avant tout une fonction d'ornement et de passage, se substituent aux espaces publics

${ }^{12}$.

Si la critique de la planification étatique et décontextualisé a favorisé la pratique du projet, ancré dans un territoire urbain, paradoxalement, les discours homogénéisant de la ville durable et numérique ont conduit à de nouvelles normes urbaines qui se déclinent localement à travers les projets urbains, eux-mêmes encouragés par des politiques renationalisées (rénovation urbaine, écoquartiers, écocités). Ils conduisent à une normalisation locale, par des conceptions de l'urbanisme de projets codifiés notamment par la technique. Ils ne permettent pas d'aborder les phénomènes de métropolisation et la diversification des phénomènes et mouvements urbains. Des villes s'étendent, s'étalent,

tandis que d'autres rétrécissent. Des quartiers sont aménagés tandis que d'autres se déploient informellement. Si la pensée rationnelle est remise en cause, et ce au profit de discours sur le local, les conceptions technico-normatives apparaissent en rupture avec les évolutions urbaines à l'œuvre, que le développement durable et numérique lui-même induit en partie. Elles conduisent au contraire à éloigner les nouveaux quartiers aménagés des pratiques et modes de vie urbains. 


\section{Une ville numérique et durable au défi des pratiques urbaines}

Les usages, sociabilités urbaines sont en pleine mutation. En effet, les citadins des

${ }^{15}$. La vie relationnelle se déploie à la fois dans la proximité (ex.flash mob

) et mondialement (réseaux). Chaque individu peut se singulariser et choisir ses groupes d'appartenance. Il peut aussi se laisser porter par une sociabilité transitive - les amis de mes amis sont mes amis

16-, vivre des formes de co-présence réelle et virtuelle, à des rythmes diversifiés.

Les relations entre les lieux et les hommes deviennent multiples. Les pratiques urbaines se superposent tant spatialement (poly-topiques), socialement (poly-appartenance) que temporellement (passé, présent et futur se croisent). Il y a un agrandissement du monde et un réinvestissement local. On observe une disjonction entre vie sociale et vie urbaine s'accroît, une grande part des citoyens maitrisant les technologies numériques.

L'évolution des modes d'être urbains restent peu considérée, les politiques urbaines voyant la technique comme réponse aux problèmes environnementaux et économiques.

Elle conduit à prescrire des savoirs faire numériques et des comportements écologiquement responsables. Autrement dit, elle invite l'individu à être en mesure de se conformer à la technique plutôt que de favoriser la multiplication de ses expériences spatiales et sociétales. 


\section{Ville numérique et durable au défi de la métropole mortelle}

Les temporalités urbaines tendent également à se fragmenter. Les rythmes urbains se superposent. Les informations sur le passé ou le futur traversent le présent en continu. Les villes elles-mêmes ne sont plus liées à une évolution chronologique. Elles décroissent, meurent tandis que d'autres émergent, entrent dans une course du mouvement perpétuel comme présent. Or, l'essor numérique et durable peine à s'adapter à cette transformation même s'il aborde les temporalités humaines de manière distinctes.

Les politiques environnementales prônent un futur à protéger au présent et valorisent les héritages. En ce sens, elle introduit les notions de réversibilité, d'adaptabilité, de mutabilité de la ville

17

. La transformation progressive est devenue un horizon urbain au détriment du projet achevé et immuable que pouvait représenter par exemple le grand ensemble. Pour autant, elle reste fortement liée à l'idée de cycles urbains comme en témoigne notamment le recyclage urbain (réemploi de matériaux, de savoirs faires, des héritages avant qu'ils ne

soient recyclés à leur tour). La question du devenir sans limite reste posée, loin d'une réversibilité liée à un contexte d'incertitude, offrant une possibilité de réviser ses choix en continu.

L'innovation numérique se centre elle sur le temps réel, qu'elle intensifie d'informations.

Le rythme s'accélère, au point d'être de moins en moins compatible avec la nature du temps humain. La temporalité présente domine les liens aux passés et aux futurs. Cette tendance à la précipitation accroît la compétition entre les villes pour se doter de réseaux numériques de plus en plus performants et rapides, étant devenus un des moteurs de l'innovation économique. Derrière les idéaux de croissance et d'innovations techniques, s'impose progressivement une société réduite au présent, où les être doivent développer des dons d'ubiquité pour vivre plusieurs présents simultanément. Dans les deux situations, la vie urbaine tend à se déconnecter du temps humain, l'une se projetant dans un futur, l'autre accélérant le temps présent. On assisterait à un éclatement de l'unité de temps, d'espace lié à une société.

\section{Vers une relation écologique entre les hommes et la technique ?}

Dans ce contexte, les ambitions prometteuses des villes numérique et durable demandent à être mieux appréhendées conceptuellement et opérationnellement. Elles appellent à être contextualisées dans les dynamiques métropolitaines. Elles impliquent d'être affranchies du mythe techniciste solutionniste qui ne peut suffire à appréhender la complexité de l'univers urbain actuel. En effet, ce mythe présente de nouveaux risques physiologiques (exposition aux ondes par exemple) urbains (remise en causes des sphères publiques), sociétaux (accélération des rythmes de vie, communauté à distance, etc.) sans s'interroger sur l'apport que ces innovations pourraient constituer pour les êtres qui 
La technique est pensée comme un système, qui s'imposerait à nous. Elle impose une grille de lecture urbaine qui se détache des usages, sociabilités, investissements humains.

Elle peine à dépasser les approches solutionnistes dans ce monde urbain devenu intelligent et durable. Elle devient alors normative et s'érige en modèle urbain avec son arsenal d'outils (référentiels, labels, indicateurs de performance, etc.), à même de servir de légitimation professionnelle. Elle pourrait pourtant être appréhendée autrement et contribuer aux évolutions urbaines, temporelles et sociétales actuelles. Elle impliquerait alors de dépasser les visons évolutionnistes et technologiques de la ville et d'accorder une place à la représentation, à l'interprétation, à l'expérience individuelle et sociétale des hommes.

\section{La technique support d'expériences sensibles des métropoles}

Avec les nouvelles technologies, les dimensions spatiales, sociales et temporelles des villes, historiquement strictement liées, se séparent en des strates de perceptions et d'expériences fragmentées. Si plutôt que de regarder les innovations du point de vue des politiques publiques nous les abordons du point de vue des individus, il nous semble que de nouvelles pistes apparaissent pour dépasser la vision solutionniste de la ville technologique durable et numérique. L'appréhension métropolitaine a de fait évolué. Elle est devenue cinétique, fugace, mouvante, fragmentée, diachronique.et non plus statique et chronologique. Chacun peut expérimenter des espaces urbains augmentés et durables ( Google map

, énergie renouvelable, transports doux, etc.). Les réalités techniques, perceptives et objectives se superposent, s'entremêlent, s'hybrident, et affectent notre rapport aux lieux 18

. Cette réalité mixte pour reprendre la fameuse expression de Paul Milgram et Fumio Kisgino

(1994) implique une nouvelle appréhension de la ville technique que celle considérée pour le progrès de l'homme ou la préservation de la planète. Elle ouvre vers une diversification d'expériences sensibles des lieux et par extension des métropoles.

Ne pourrait-elle pas être un levier d'expérience urbaine à l'interface des multiples strates

du réel que chacun expérimente, habite et compose à sa façon ? Comme on l'a vu, les niveaux d'expériences des métropoles se diversifient. Les pratiques urbaines s'hybrident

20

. Le statut des lieux évolue. Si les réseaux sociaux constituent des espaces virtuels, ils mettent au premier plan l'appartenance sociale à un groupe plutôt qu'à un lieu. La dynamique relationnelle prime et l'expérience urbaine est devenue polytopiques. Comment les métropoles numériques et durables pourraient favoriser des appartenances multiples tout en garantissant une unité sociétale?

Parallèlement, la technologie envahi l'appréhension des lieux. La virtualité est un univers idéel, telle que l'avait introduit Antonin Artaud avec les représentations virtuelles

. L'hybridation des univers techniques, réels et imaginaires (invasion des puces et des procédés tactiles ou scannant le mouvement) ne cesse de croître. Les liens sont marqués de liens hypertextes d'appréciation (on fréquente les lieux repérer par des « 
like

", etc.). Or, si la ville est faite de signes et significations, elle se métamorphose avec ces repères géolocalisés liés aux commentaires de chacun

22

. Elle crée de nouvelles images, évocations subjectives, suggestives et imaginaires des métropoles. Cette réalité augmentée d'information crée un réel aux multiples strates, en mille feuilles. Comment dès lors la métropole peut être un lieu d'expériences plurielles tout en créant des paysages et espaces urbains communs?

Aborder la technique du point de vue de la perception individuelle et collective ne seraitce pas une perspective pour concevoir des métropoles moins technicistes et plus respectueuses des hommes? Ne faudrait-il pas alors observer la technique d'un point de vue phénoménologique, c'est-à-dire en considérant que la technique change l'apparition des phénomènes 23

? Celle-ci rendrait alors visible le monde technologique et inviterait à des approches phénoménotechniques

24

. Elle ferait apparaître le réel et ses différentes strates, comme tout objet, toujours liée à une culture, un imaginaire. En effet, la technique n'existe pas en soi. Elle est autant réelle qu'une interprétation, une représentation, une reproduction mentale d'une perception,

d'une sensation précédemment éprouvée. Elle est une forme sous laquelle un objet est perçu, mais aussi une manifestation, une métaphore de l'espace abstrait. Elle peut devenir alors un enjeu d'appréhension du monde. Par exemple, le numérique apparaît et se donne au sujet plus qu'il ne résulte de l'interaction entre le sujet et l'objet

25

. Elle exprime cette totalité visible et invisible. Elle fixe le temps qui file, elle inscrit une mémoire et des visions futures. Elle permet de se situer dans un univers mouvant. Elle révèle le monde spatial et temporel infini, mouvant, éclaté et fragmenté.

Elle permet d'aborder autrement les paysages, ambiances, espaces urbains comme médium entre le réel et l'idéel. À la Renaissance, le paysage découpe dans la nature un tableau, et change la perception du monde. Au 19

siècle, la remise en cause de la géométrie d'Euclide fait évoluer la perception terrestre comme aujourd'hui, la théorie quantique, qui énonce qu'une particule existe en plusieurs endroits simultanément, bouleverse la vision du temps et de l'espace. Cette vision n'est pas perceptible par l'homme mais est symbolisées par la machine, l'énergie, le paysage technologique

. La technique pourrait créer une brèche pour se raccrocher soi et collectivement à ce monde incertain et porteur de menaces écologiques et sociales. Elle pourrait plus encore participer à l'essor de métropole réconciliant les dimensions rationnelles et sensibles des espaces urbains. Au delà de la 
Senseable city Lab, (Carlo Ratti, Directeur duMassachusetts Institute of Technology

) à l'interface entre les villes, le numérique et les gens, la métropole sensible intégrerait les différentes formes techniques comme support d'expériences propres à chacun. Il s'agirait selon nous de considérer autant les sens donnés aux technologiques que les interprétations, représentations et imaginaires, voire les émotions et sensations suscitées. Autrement dit, une évolution des cultures perceptives, des possibilités de sentir et de se représenter le monde à l'interface du subjectif, de l'objectif, de l'idéel et du technologique, serait à inventer.

La technique considérée du point de vue phénoménologique pourrait alors s'attacher à révéler le monde, à susciter des expériences, des appréhensions inédites du monde qu'elle souhaite préserver, représenter, rendre plus performant. L'image de l'univers pourrait être approchée, au-delà de son infinitude, de sa fragilité, de son éclatement en mille strates. Tout un chacun pourrait l'éprouver, en prendre conscience et la ménager à sa manière. Tout individu pourrait agencer ses ressentis, être libre de ses initiatives pour le devenir urbain. Elle pourrait redonner une place à l'individu, non pas en tant qu'être conforme à la norme technique mais comme être en mesure d'inventer une nouvelle relation médiatisée aux lieux, au monde, à l'univers. Elle aurait alors un caractère durable, dans la mesure où elle pourrait être réinventée en continu par les sociétés humaines et les individus, notamment dans les interrelations entre les strates du réel, entre soi et les autres, entre les hommes et le monde.

\section{BIBLIOGRAPHIE}

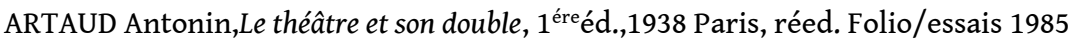

BACHELARD Gaston,L'Expérience de l'espace dans la physique contemporaine, Paris, Alcan, 1937

BADIE Bertrand,La fin des territoires - Essai sur le désordre international et sur l'utilité sociale du respect , Paris, Fayard, 1997

BAILLEUL Hélène, “ Les nouvelles formes de la communication autour des projets urbains : modalités, impacts, enjeux pour un débat participatif. Analyse du rôle des images dans le débat participatif autour de deux projets urbains en France »,

Métropoles, Paris, 2008

BAILLY Emeline, “ De la ville nature à la ville paysage ",Projet de recherche Fabrique de la ville , Paris, CSTB, 2014

BAILLY Emeline (dir.), DURET Hervé, TUAL Mélanie, “ Quels apports des maquettes numériques urbaines dans les projets urbains durables?",

Programme de recherche Ville Numérique (SONVN), Paris, CSTB, 2013

BELZITI Daniela (dir.), BAILLY Emeline, SOULA Julien, TUAL Mélanie, “L'enjeu du numérique pour la ville durable»,

Programme de recherche Fabrique de la ville, Paris, CSTB, 2014 
BERDOULAY Vincent, C. Da Costa Gomes Paulo et Lolive Jacques (sous la direction), L'espace public à l'épreuve : régressions et émergences

,Éd. Maison des Sciences de l'Homme Aquitaine, 2004

CRABTREE Andy et RODDEN Tom,

Hybrid Ecologies : Understanding Cooperative Interaction in Emerging Physical-digital Environments , Springer-Verlag,London, 2007

ECHENNE Fabien, La ville 2.0, complexe et familière, la fabrique des possibles, Paris, FYP Editions, 2008

LEDRUT Raymond,Les images de la ville, Paris, Éd. Anthropos, 1973

LEVY Jacques, «Penser la ville : un impératif sous toutes les latitudes »,revue Cemoti, $\mathrm{n}^{\circ} 24$, Métropoles et métropolisation, Paris, février 2005

LEVY Jacques, entretien inSylvain ALLEMAND (dir), Villes 2.0. La Recherche Urbaine à l'heure de la ville 2.0 , Étude menée dans le cadre du programme « Ville $2.0 »$, FING. Disponible à l'adresse : [ http://doc.openfing.org/Villes2/Recherche_urbaine_villes2.pdf]

MAGNAGHI Alberto,La bio région urbaine. Petit traité sur le territoire bien commun , Paris, Eterotopia Rhizome, 2014

MATTHEY Laurent, « Urbanisme fictionnel : l'action urbaine à l'heure de la société du spectacle ", Métropolitiques,Paris, 28 octobre 2011

MARZLOFF Bruno.« Le numérique urbain face au développement durable »,Techni.Cités194, Paris, septembre 2010

MILGRAM Paul et KISGINO Fumio, « Taxonomy of Mixed Reality Visual Displays ", SPIE vol. 2351, Telemanipulator and Telepresence Technologies, Tonroto, 1994

RATTI Carlo,SENSEable City Laboratory, Massachusetts Institute of Technology, 2014

SASSEN Saskia,Global city, New York, London, Tokyo, Princeton, Princeton University Press, 1991

SCHERRER Franck, VANIER Martin (dir.),Villes, territoires, réversibilité, Paris, Hermann, 2013

SIMMEL Georg,Études et formes de la socialisation, PUF, Paris, 2010

SOULA J., BAILLY E., DURET H., TUAL M, et Al. «La maquette numérique à l'échelle urbaine » in BIM \& maquette numérique : Pour l'architecture, le bâtiment et la construction sous la direction de Eric LEBEGUE et Olivier CELNIK, Cstb, Eyrolles, Juin 2014

TURNER Fred,

Aux sources de l'utopie numérique : De la contre culture à la cyberculture, Stewart Brand un homme d'influence

, Trad. de l'anglais par Laurent Vannini, Caen, C\&F Éd., 2012

VIAL Stéphane,L'être et l'écran, Paris, PUF, 2013.

URLBERGER Andrea,Paysage technologique, théories et pratiques autour du GPS , EdNM/Ciren, Université Paris 8, sept. 2007. Disponible à l'adresse : [ http://www.ciren.org/ciren/laboratoires/Paysage_Technologique/index.html]

ZREIK Khaldoun, (dir.),Villes hybrides et enjeux de l'aménagement des urbanités numériques , Paris, Europia Productions, 2012 


\section{NOTES}

1.

BELZITI Daniela (dir.), BAILLY Emeline, SOULA Julien, TUAL Mélanie, « L'enjeu du numérique pour la ville durable », Programme de recherche Fabrique de la ville, Paris, CSTB, 2014.

2.

SASSEN Saskia,Global city

, New York, London, Tokyo, Princeton, Princeton University Press, 1991.

3. LEVY Jacques, «Penser la ville : un impératif sous toutes les latitudes», revueCemoti, $\mathrm{n}^{\circ} 24$, Métropoles et métropolisation, Paris, février 2005.

4.

Rome, Istanbul, ou Karbala sont des villes influentes d'un point de vue religieux et patrimonial mais elles ne sont pas considérées comme des «

global cities » faute de puissance économique.

5.

Le développement durable est défini comme « un développement qui répond aux besoins du présent sans compromettre la capacité des générations futures à répondre aux leurs ».

6. BERDOULAY Vincent, C. DA COSTA Gomes Paulo et LOLIVE Jacques (dir.),

L'espace public à l'épreuve : régressions et émergences

,Éd. Maison des Sciences de l'Homme Aquitaine, 2004.

7.

BELZITI Daniela (dir.), BAILLY Emeline, SOULA Julien, TUAL Mélanie, « L’enjeu du numérique pour la ville durable », op.cit.

8.

CRABTREE Andy, RODDEN Tom,

Hybrid ecologies: understanding cooperative interaction in emerging physical-digital environments , Springer-Verlag, London, 2007.

9.

BADIE Bertrand,

La fin des territoires. Essai sur le désordre international et sur l'utilité sociale du respect

, Paris, Fayard, 1997.

10.

TURNER Fred,

Aux sources de l'utopie numérique : De la contre culture à la cyberculture, Stewart Brand un homme

d'influence

, Trad. de l'anglais par Laurent Vannini, Caen, C\&F Éd., 2012.

11. MAGNAGHI Alberto,La bio région urbaine. Petit traité sur le territoire bien commun , Paris, Eterotopia Rhizome, 2014.

12. BAILLY Emeline, « De la ville nature à la ville paysage ",projet de recherche Fabrique de la ville , PARIS, CSTB, 2014.

13. SIMMEL Georg,Etudes et formes de la socialisation, coll. Sociologie, PUF, Paris, 2010.

14. ECHENNE Fabien, La ville 2.0, complexe... et familière, la fabrique des possibles, FYP Editions, 2008.

15. Ibidem.

16.

LEVY Jacques, entretien inALLEMAND, Sylvain (dir),

Villes 2.0. La Recherche Urbaine à l'heure de la ville 2.0

,Étude menée dans le cadre du programme « Ville 2.0 », FING, [http://doc.openfing.org/Villes2/ Recherche_urbaine_villes2.pdf]

17.

SCHERRER Franck, VANIER Martin (dir.),Villes, territoires, Réversibilité, Paris, Hermann, Coll. Société, 2013.

18. ZREIK Khaldoun, (dir.),Villes hybrides et enjeux de l'aménagement des urbanités numériques , Europia Productions, 2012. 
19.

MILGRAM Paul, KISGINO Fumio, « Taxonomy of Mixed Reality Visual Displays », SPIE Vol. 2351, Telemanipulator and Telepresence Technologies, Toronto, 1994.

20.

LAUDATI Patricia, « Nouvelles perceptions et nouveaux usages urbains par les technologies numériques, le point de vue des habitants ", i

$n$ Khaldoun ZREIK, (dir.),Villes hybrides et enjeux de l'aménagement des urbanités numériques

, Europia Productions, 2012.

21. ARTAUD Antonin,Le théâtre et son double, Paris, 1938, réed. Folio/essais 1985.

22. LEDRUT Raymond, Les images de la ville. Paris, Ed. Anthropos, 1973.

23. Phénomène : expérience par la perception.

24. BACHELARD Gaston,L'Expérience de l'espace dans la physique contemporaine, Paris, Alcan, 1937.

25. VIAL Stéphane,L'être et l'écran, Paris, PUF, 2013.

26. URLBERGER, Andrea,Paysage technologique, théories et pratiques autour du GPS

, EdNM/Ciren, Université Paris 8, sept. 2007.

\section{RÉSUMÉS}

L'enjeu du numérique apparaît de plus en plus prégnant dans les projets urbains durables. Il s'apparente parfois à une nouvelle marque de fabrique urbaine : ville numérique, ville intelligente,connected city

. Notre hypothèse est qu'il traduit un nouvel idéal de ville technologique durable. De fait, la conception urbaine a souvent évolué grâce à l'introduction de technologies nouvelles. La maîtrise agraire et la canalisation de l'eau ont permis l'édification des premières villes, l'édification des routes, leurs mises en réseaux commerciales, l'industrialisation, le développement de la ville moderne fonctionnaliste (rails, infrastructure, zoning

, etc.). Elle s'est accompagnée des visions utopistes successives où la technologie apparaissait comme l'emblème du progrès humain. À présent, avec le développement durable, l'ambition technique, en particulier environnementale et numérique, doit au contraire préserver la planète, les hommes et les civilisations. La technologie change ainsi de statut. Elle n'est plus pour le progrès de l'homme, mais pour la pérennité du monde. Comment, dès lors, ce renversement de paradigme impacte-t-il la culture technique héritée et la

conception urbaine actuelle ? En quoi ces techniques environnementales visant la maîtrise d'énergie, de l'eau, la protection des ressources... et celles numériques visant la création de réseaux intelligents, nouveaux services urbains, etc., permettent-elles de concevoir une ville durable ? Pour répondre à ces questions, nous chercherons à comprendre ce qui fonde la ville durable et numérique pour les politiques publiques afin de questionner les idéaux technologiques urbains associés. Après une analyse de la mobilisation des technologies dans les discours actuels des professionnels de la ville, nous questionnerons la possibilité d'un changement de paradigme de la technique pour des métropoles au service de la durabilité de la planète mais aussi offrant des expériences à la fois sensibles et objectives des lieux urbains. 
Sustainable urban projects value more and more the digital challenge. Digital city, intelligent

city, connected city become an urban brand. Our hypothesis is that it conveys a new ideal of sustainable and digital city. Indeed, urban design has often evolved thanks to the introduction of new technologies. The command of agrarian production, canal digging and transportation of water led to the construction of early cities and roads, their connections in commercial networks, industrialization, and the development of the modern and functional city (railways, infrastructure, zoning, etc.). The conception was also accompanied by successive utopian visions, which consider technology as the symbol of human progress. Now, with sustainable development, the technical ambition, particularly environmental and digital must preserve the planet, humanity and civilization. Therefore, the status of technology changes. It no longer aims at human progress, but at ensuring the durability of the world. How does this paradigm shift affect the inherited technical culture and the current urban design? In which aspects do these environmental techniques aiming at energy and water management, resources protection, and digital technologies aiming at the creation of intelligent networks, shape the concept of a sustainable city? To answer to these questions, we will try to understand what founds the sustainable and digital city in public policies, in order to inquire the urban technological ideals. After an analysis of technologies mobilization in present discussions among urban professionals, we inquire the possibility of a technology paradigm shift, for metropolises in favor of the planet sustainability, but also offering simultaneously substantial and objective experiences of urban

places.

\section{INDEX}

Mots-clés : Histoire des techniques, technologies urbaines, villes numériques, villes durables, villes sensibles

Keywords : History of technology, urban technology, digital cities, sustainable cities, sensitive cities

\section{AUTEUR}

\section{ÉMELINE BAILLY}

Docteur en urbanisme, Émeline Bailly est chercheur au CSTB/Université Paris Est sur la conception urbaine durable et les concepts de paysage, d'espace public et d'urbanité. Elle conduit parallèlement des missions et programmes de recherches urbaines à l'échelle internationale (New York, Téhéran, Rabat, Phnom Penh, etc.) et intervient régulièrement dans divers instituts d'urbanisme et écoles d'architecture en France et à l'étranger (Fordham University, Yale Universityà New York, INAU à Rabat, etc.). 